\title{
Orthogonal polynomials with respect to generalized Jacobi measures
}

\author{
Tivadar Danka
}

A thesis submitted for the degree of
Doctor of Philosophy

Supervisor: Vilmos Totik

Doctoral School in Mathematics and Computer Science University of Szeged, Bolyai Institute 
One of the first sentences of the seminal paper Extremal polynomials associated with a system of curves in the complex plane by Harold Widom is "All asymptotic formulas have refinements." The goal of this thesis is to refine, extend and establish asymptotic formulas related to orthogonal polynomials with respect to generalized Jacobi measures, i.e. measures having an algebraic singularity $\left|x-x_{0}\right|^{\alpha} d x$ somewhere in their support.

The main objects of our study are the Christoffel-Darboux kernel defined by

$$
K_{n}(z, w)=\sum_{k=0}^{n-1} p_{k}(z) \overline{p_{k}(w)},
$$

where $p_{n}$ denotes the $n$-th orthonormal polynomial with respect to $\mu$, and the Christoffel function defined by

$$
\lambda_{n}\left(\mu, z_{0}\right)=\inf _{\operatorname{deg} P<n} \int \frac{|P(z)|^{2}}{\left|P\left(z_{0}\right)\right|^{2}} d \mu(z),
$$

where the infimum is taken for all nonzero polynomials of degree $n-1$. It is a known fact that

$$
\lambda_{n}\left(\mu, z_{0}\right)=\frac{1}{K_{n}\left(z_{0}, z_{0}\right)} .
$$

The study of Christoffel functions has started in the beginning of the XXth century, one important early result is due to Gábor Szegö. He proved that if $\mu$ is a measure supported on the unit circle 
$\mathbb{T}$ which is absolutely continuous with $d \mu\left(e^{i t}\right)=w\left(e^{i t}\right) d t$ and

$$
\frac{1}{2 \pi} \int_{-\pi}^{\pi} \log w\left(e^{i t}\right) d t>-\infty
$$

holds, which is called Szegő condition, then we have

$$
\lim _{n \rightarrow \infty} \lambda_{n}(\mu, z)=\left(1-|z|^{2}\right) e^{\operatorname{Re} D(\mu, z)}, \quad|z|<1,
$$

where $D(\mu, z)$ is the Szegö function defined by

$$
D(\mu, z)=\frac{1}{2 \pi} \int_{-\pi}^{\pi} \frac{e^{i t}+z}{e^{i t}-z} \log w\left(e^{i t}\right) d t .
$$

The influence of this result can be seen in the asymptotic theory of orthogonal polynomials, but it has also served as a motivation to study Hardy spaces.

If we study the asymptotics in the points of the unit circle, we have

$$
\lim _{n \rightarrow \infty} \lambda_{n}(\mu, z)=\mu(\{z\}), \quad|z|=1,
$$

which is zero, if the measure is absolutely continuous, therefore this does not provide much useful information. In this case, the main question is to determine the exact order of asymptotics. A. Máté, P. Nevai and V. Totik proved in the seminal paper [2] that if $\mu$ is supported on the unit circle with $d \mu\left(e^{i t}\right)=w\left(e^{i t}\right) d t+d \mu_{s}\left(e^{i t}\right)$ there, then if the Szegö condition

$$
\frac{1}{2 \pi} \int_{-\pi}^{\pi} \log w\left(e^{i t}\right) d t>-\infty
$$


holds, we have

$$
\lim _{n \rightarrow \infty} n \lambda_{n}\left(\mu, e^{i t}\right)=2 \pi w\left(e^{i t}\right)
$$

for $t \in[-\pi, \pi)$ almost everywhere. A similar result was proved for measures supported on $[-1,1]$.

For measures supported on a general compact subset of the real line, the above results were extended by Totik in [6] using the polynomial inverse image method developed by him in [8]. He showed that if $\mu$ is regular in the sense of Stahl-Totik and it is supported on a compact set $\operatorname{supp}(\mu)=K$ with $d \mu(x)=$ $w(x) d x+d \mu_{s}(x)$ there, then if the local Szegö condition

$$
\int_{I} \log (w(x)) \omega_{K}(x) d x
$$

holds, where $\omega_{K}$ is the density function of the equilibrium measure, we have

$$
\lim _{n \rightarrow \infty} n \lambda_{n}(\mu, x)=\frac{w(x)}{\omega_{K}(x)}
$$

for $x \in I$ almost everywhere. Asymptotics are also established for measures supported on a set of disjoint Jordan arcs and curves. Totik proved in [7] that if $\mu$ is supported on a disjoint union of Jordan curves $\gamma$ lying exterior to each other, then if $z_{0} \in \gamma$ and $\mu$ is absolutely continuous with respect to the arc length measure $s_{\gamma}$ in a small subarc containing $z_{0}$ and $d \mu(z)=w(z) d s_{\gamma}(z)$ there for 
some continuous and strictly positive weight $w$, we have

$$
\lim _{n \rightarrow \infty} n \lambda_{n}\left(\mu, z_{0}\right)=\frac{w\left(z_{0}\right)}{\omega_{\gamma}\left(z_{0}\right)},
$$

where $\omega_{\gamma}$ again denotes the Radon-Nikodym derivative of the equilibrium measure with respect to the arc length measure $s_{\gamma}$.

If, however, $w(z)$ is not continuous or positive at the prescribed point $z_{0}$, the asymptotics in (1) does not hold anymore. The first main result of this thesis is the generalization of this result for measures exhibiting $d \mu(z)=w(z)\left|z-z_{0}\right|^{\alpha} d s_{\gamma}(z)$ behavior around $z_{0}$ for some $\alpha>-1$. Our main result in this setting is the following.

Theorem 1. Let $\gamma$ be a disjoint union of rectifiable Jordan curves lying exterior to each other and let $\mu$ be a finite Borel measure regular in the sense of Stahl-Totik with support $\operatorname{supp}(\mu)=\gamma$. Suppose that for a $z_{0} \in \gamma$, there is an open set $U$ such that $J=U \cap \gamma$ is a $C^{2}$ smooth Jordan arc and $\mu$ is absolutely continuous with respect to the arc length measure and

$$
d \mu(z)=w(z)\left|z-z_{0}\right|^{\alpha} d s_{\gamma}(z), \quad z \in J
$$

there for some $\alpha>-1$ and some weight function $w$ which is strictly positive and continuous at $z_{0}$. Then

$$
\lim _{n \rightarrow \infty} n^{\alpha+1} \lambda_{n}\left(\mu, z_{0}\right)=\frac{w\left(z_{0}\right)}{\left(\pi \omega_{\gamma}\left(z_{0}\right)\right)^{\alpha+1}} 2^{\alpha+1} \Gamma\left(\frac{\alpha+1}{2}\right) \Gamma\left(\frac{\alpha+3}{2}\right)
$$


holds, where $\Gamma(z)$ denotes the Gamma function and $\omega_{\gamma}$ again denotes the Radon-Nikodym derivative of the equilibrium measure with respect to the arc length measure $s_{\gamma}$.

Asymptotics for the Christoffel-Darboux kernel can also be studied off the diagonal. One area of interest is the so-called universality limits for random matrices, which is an intensively studied topic of mathematical physics, having several applications even outside mathematics.

For measures supported on $[-1,1]$, a new approach for universality limits was developed by D. S. Lubinsky in the seminal papers [3] [4] [5]. In [3] it was shown that if $\mu$ is a finite Borel measure supported on $[-1,1]$ which is regular in the sense of Stahl-Totik and absolutely continuous with $d \mu(x)=w(x) d x$ in a neighbourhood of $x_{0} \in(-1,1)$, where $w(x)$ is also continuous and strictly positive, then the universality limit

$$
\lim _{n \rightarrow \infty} \frac{\widetilde{K}_{n}\left(x_{0}+\frac{a}{\widetilde{K}_{n}\left(x_{0}, x_{0}\right)}, x_{0}+\frac{b}{\widetilde{K}_{n}\left(x_{0}, x_{0}\right)}\right)}{\widetilde{K}_{n}\left(x_{0}, x_{0}\right)}=\frac{\sin \pi(b-a)}{\pi(b-a)}
$$

holds, where $\widetilde{K}_{n}(x, y)=\sqrt{w(x) w(y)} K_{n}(x, y)$ denotes the normalized Christoffel-Darboux kernel. The terminology universality limit comes from the fact that inside the limit we have a quantity which is heavily dependent on the measure, but after taking the limit, it is independent of it. Before this result of Lubinsky, 
analiticity of the weight function was required on the whole support $[-1,1]$, therefore this was a large step ahead.

When the measure exhibits singular behavior at the prescribed point $x_{0}$, for example it behaves like $\left|x-x_{0}\right|^{\alpha} d x$ for some $\alpha>-1$, it no longer shows the same behavior and instead of the sinc kernel, something else appears. Generalized Jacobi measures of the form

$$
d \mu(x)=(1-x)^{\alpha}(1+x)^{\beta} h(x) d x, \quad x \in[-1,1],
$$

where $h(x)$ is positive and analytic, were studied by A. B. J. Kuijlaars and M. Vanlessen in [1]. Using Riemann-Hilbert methods, they showed that

$$
\lim _{n \rightarrow \infty} \frac{1}{2 n^{2}} \widetilde{K}_{n}\left(1-\frac{a}{2 n^{2}}, 1-\frac{b}{2 n^{2}}\right)=\mathbb{J}_{\alpha}(a, b)
$$

uniformly for $a, b$ in compact subsets of $(0, \infty)$, where $\mathbb{J}_{\alpha}(a, b)$ is the so-called Bessel kernel defined as

$$
\mathbb{J}_{\alpha}(a, b)=\frac{J_{\alpha}(\sqrt{a}) \sqrt{b} J_{\alpha}^{\prime}(\sqrt{b})-J_{\alpha}(\sqrt{b}) \sqrt{a} J_{\alpha}^{\prime}(\sqrt{a})}{2(a-b)}
$$

and $J_{\alpha}(x)$ denotes the Bessel function of the first kind and order $\alpha$. This was extended by Lubinsky in [4]. He proved that if $\mu$ is a finite Borel measure supported on $[-1,1]$ which is absolutely continuous on $[1-\varepsilon, 1]$ for some $\varepsilon>0$ with

$$
d \mu(x)=w(x)|x-1|^{\alpha}, \quad x \in[1-\varepsilon, 1]
$$


there, where $w(x)$ is strictly positive and continuous at 1 , then

$$
\lim _{n \rightarrow \infty} \frac{1}{2 n^{2 \alpha+2}} K_{n}\left(1-\frac{a}{2 n^{2}}, 1-\frac{b}{2 n^{2}}\right)=\mathbb{J}_{\alpha}^{*}(a, b)
$$

holds, where $\mathbb{J}_{\alpha}^{*}(a, b)=\frac{\mathbb{J}_{\alpha}(a, b)}{a^{\alpha / 2} b^{\alpha / 2}}$ is the entire version of the Bessel kernel. It was also shown by Lubinsky in [5] that if $K$ is a compact subset of the real line and $x_{0} \in K$ is a right endpoint of $K$ (i.e. there exists an $\varepsilon>0$ such that $K \cap\left(x_{0}, x_{0}+\varepsilon\right)=\varnothing$ ), then if $\mu$ is a finite Borel measure with $\operatorname{supp}(\mu)=K$ which is absolutely continuous in a small left neighbourhood of $x_{0}$ behaving like $d \mu(x)=\left|x-x_{0}\right|^{\alpha} d x$ there for some $\alpha>-1$, then

$$
\lim _{n \rightarrow \infty} \frac{K_{n}\left(x_{0}-a \eta_{n}, x_{0}-a \eta_{n}\right)}{K_{n}\left(x_{0}, x_{0}\right)}=\frac{\mathbb{J}_{\alpha}^{*}(a, a)}{\mathbb{J}_{\alpha}^{*}(0,0)}
$$

for all $a \in[0, \infty)$ implies

$$
\lim _{n \rightarrow \infty} \frac{K_{n}\left(x_{0}-a \eta_{n}, x_{0}-b \eta_{n}\right)}{K_{n}\left(x_{0}, x_{0}\right)}=\frac{\mathbb{J}_{\alpha}^{*}(a, b)}{\mathbb{J}_{\alpha}^{*}(0,0)}
$$

uniformly for $a, b$ on compact subsets of the complex plane, where the sequence $\eta_{n}$ is $\eta_{n}=\left(\mathbb{J}_{\alpha}^{*}(0,0) / K_{n}\left(x_{0}, x_{0}\right)\right)^{1 /(\alpha+1)}$. In such a general setting, it was not known if (4) holds.

In the second main part of the thesis, we show that (4) does indeed hold, hence (5) also holds as well. On the other hand, we also aim to establish universality limits in the case when the singularity is located in the interior of the support rather than at the hard edge. 
In order to express universality limits for measures exhibiting power-type singularity in the interior of its support, we define the kernel function for $a, b \in \mathbb{R}$ by

$$
\mathbb{L}_{\alpha}(a, b)=\frac{\sqrt{a b}}{2(a-b)}\left(J_{\frac{\alpha+1}{2}}(a) J_{\frac{\alpha-1}{2}}(b)-J_{\frac{\alpha+1}{2}}(b) J_{\frac{\alpha-1}{2}}(a)\right)
$$

if $a, b \geq 0$,

$$
\mathbb{L}_{\alpha}(a, b)=\frac{\sqrt{a(-b)}}{2(a-b)}\left(J_{\frac{\alpha+1}{2}}(a) J_{\frac{\alpha-1}{2}}(-b)+J_{\frac{\alpha+1}{2}}(-b) J_{\frac{\alpha-1}{2}}(a)\right)
$$

if $a \geq 0, b<0$, and $\mathbb{L}_{\alpha}(a, b)=\mathbb{L}_{\alpha}(-a,-b)$ otherwise, where $J_{\nu}(x)$ denotes the Bessel functions of the first kind and order $\nu$. Since $J_{\nu}(z)=z^{\nu} G(z)$ where $G(z)$ is an entire function, we can define the entire version of the kernel function for arbitrary complex arguments as

$$
\mathbb{L}_{\alpha}^{*}(a, b)=\frac{\mathbb{L}_{\alpha}(a, b)}{a^{\alpha / 2} b^{\alpha / 2}}, \quad \mathbb{L}_{\alpha}^{*}(a)=\frac{\mathbb{L}_{\alpha}(a, a)}{a^{\alpha}}, \quad a, b \in \mathbb{C} .
$$

Our main results are the following four theorems. The first two deals with the asymptotics of Christoffel functions when the power type singularity is in the interior (in other words, in the bulk) or at an endpoint (in other words, at the hard edge). The last two theorems are concerned with universality limits in the same cases.

Theorem 2. Let $\mu$ be a finite Borel measure which is regular in the sense of Stahl-Totik and suppose that $\mu$ is supported on a compact set $K=\operatorname{supp}(\mu)$ on the real line. Let $x_{0} \in \operatorname{int}(K)$ be a point 
from the interior of its support and suppose that on some small interval $\left(x_{0}-\varepsilon_{0}, x_{0}+\varepsilon_{0}\right)$ containing $x_{0}$, the measure $\mu$ is absolutely continuous with

$$
d \mu(x)=w(x)\left|x-x_{0}\right|^{\alpha} d x, \quad x \in\left(x_{0}-\varepsilon_{0}, x_{0}+\varepsilon_{0}\right)
$$

there for some $\alpha>-1$ and $\alpha \neq 0$, where $w$ is strictly positive and continuous at $x_{0}$. Then

$$
\lim _{n \rightarrow \infty} n^{\alpha+1} \lambda_{n}\left(\mu, x_{0}+\frac{a}{n}\right)=\frac{w\left(x_{0}\right)}{\left(\pi \omega_{K}\left(x_{0}\right)\right)^{\alpha+1}}\left(\mathbb{L}_{\alpha}^{*}\left(\pi \omega_{K}\left(x_{0}\right) a\right)\right)^{-1}
$$

holds uniformly for a in compact subsets of the real line, where $\mathbb{L}_{\alpha}^{*}(\cdot)$ is defined by $(6)$.

The analogue for the edge is the following.

Theorem 3. Let $\mu$ be a finite Borel measure which is regular in the sense of Stahl-Totik and suppose that $\mu$ is supported on a compact set $K=\operatorname{supp}(\mu)$ on the real line. Let $x_{0} \in K$ be a right endpoint of $K$ (i.e. $K \cap\left(x_{0}, x_{0}+\varepsilon_{1}\right)=\varnothing$ for some $\left.\varepsilon_{1}>0\right)$ and assume that on some interval $\left(x_{0}-\varepsilon_{0}, x_{0}\right]$ the measure $\mu$ is absolutely continuous with

$$
d \mu(x)=w(x)\left|x-x_{0}\right|^{\alpha} d x, \quad x \in\left(x_{0}-\varepsilon_{0}, x_{0}\right]
$$

there for some $\alpha>-1$, where $w$ is strictly positive and left continuous at $x_{0}$. Then

$\lim _{n \rightarrow \infty} n^{2 \alpha+2} \lambda_{n}\left(\mu, x_{0}-\frac{a}{2 n^{2}}\right)=\frac{w\left(x_{0}\right)}{M\left(K, x_{0}\right)^{2 \alpha+2}}\left(2^{\alpha+1} \mathbb{J}_{\alpha}^{*}\left(M\left(K, x_{0}\right)^{2} a\right)\right)^{-1}$ 
holds uniformly for a in compact subsets of $[0, \infty)$, where $\mathbb{J}_{\alpha}^{*}(\cdot)$ is the Bessel kernel defined by (3) and $M\left(K, x_{0}\right)$ is defined by

$$
M\left(K, x_{0}\right)=\lim _{x \rightarrow x_{0}-} \sqrt{2} \pi\left|x-x_{0}\right|^{1 / 2} \omega_{K}(x) .
$$

By symmetry, there is a similar result for left endpoints. From the asymptotics for Christoffel functions we obtain universality limits.

Theorem 4. With the assumptions of Theorem 2, we have

$$
\lim _{n \rightarrow \infty} \frac{K_{n}\left(x_{0}+\frac{a}{n}, x_{0}+\frac{b}{n}\right)}{K_{n}\left(x_{0}, x_{0}\right)}=\frac{\mathbb{L}_{\alpha}^{*}\left(\pi \omega_{K}\left(x_{0}\right) a, \pi \omega_{K}\left(x_{0}\right) b\right)}{\mathbb{L}_{\alpha}^{*}(0,0)}
$$

uniformly for $a, b$ in compact subsets of the complex plane.

Theorem 5. With the assumptions of Theorem 3, we have

$$
\lim _{n \rightarrow \infty} \frac{K_{n}\left(x_{0}-\frac{a}{2 n^{2}}, x_{0}-\frac{b}{2 n^{2}}\right)}{K_{n}\left(x_{0}, x_{0}\right)}=\frac{\mathbb{J}_{\alpha}^{*}\left(M\left(K, x_{0}\right)^{2} a, M\left(K, x_{0}\right)^{2} b\right)}{\mathbb{J}_{\alpha}^{*}(0,0)} .
$$

uniformly for $a, b$ in compact subsets of the complex plane.

Again by symmetry, there is a similar result for left endpoints.

The proofs of the main results are done in several steps. First, we study the measures $\mu_{\alpha}^{b}$ and $\mu_{\alpha}^{e}$ supported on $[-1,1]$ and defined by

$$
d \mu_{\alpha}^{b}(x)=|x|^{\alpha}, \quad x \in[-1,1]
$$


and

$$
d \mu_{\alpha}^{e}(x)=|x-1|^{\alpha}, \quad x \in[-1,1]
$$

Using the Riemann-Hilbert method, we shall prove (7) for $\mu_{\alpha}^{b}$ and (8) for $\mu_{\alpha}^{e}$, which shall serve as a model case for our investigations about measures supported on the real line. Then we transform some of the results to obtain Theorem 1 for the measure $\mu_{\alpha}^{\mathbb{T}}$ defined by

$$
d \mu_{\alpha}^{\mathbb{T}}\left(e^{i t}\right)=\left|e^{i t}-i\right|^{\alpha} d t, \quad t \in[-\pi, \pi) .
$$

After this, we shall transform these model cases using the polynomial inverse image method of Totik, to obtain Theorems 1, 2 and 3 in their full generailty. After these, we prove Theorems 4 and 5 using the normal family approach of Lubinsky.

\section{The thesis is based on the following publications.}

[1] T. DAnkA and V. TотіK, Christoffel functions with power type weights, to appear in J. Eur. Math. Soc., available at arXiv with identifier arXiv:1504.03968

[2] T. DANKA, Universality limits for generalized Jacobi measures, submitted for consideration for publication, available at arXiv with identifier arXiv:1605.04275 


\section{References}

[1] A. B. J. KuiJlaars and M. Vanlessen, Universality for eigenvalue correlations at the origin of the spectrum, Comm. Math. Phys., 243(2003), 163-191

[2] A. MÁté, P. Nevai and V. Totik, Szegő's extremum problem on the unit circle, Ann. of Math., Vol 134, No. 2. (1991), 433-453

[3] D. S. Lubinsky, A new approach to universality limits involving orthogonal polynomials, Ann. of Math., 170(2009), 915-939

[4] D. S. Lubinsky, A new approach to universality at the edge of the spectrum, Contemporary Mathematics (60th birthday of Percy Deift), 458(2008), 281-290

[5] D. S. Lubinsky, Universality limits at the hard edge of the spectrum for measures with compact support, Int. Math. Res. Not. 2008

[6] V. Tотік, Asymptotics for Christoffel functions for general measures on the real line, J. D'Analyse Math., 81(2000), 283303 
[7] V. Tотік, Christoffel functions on curves and domains, Trans. Amer. Math. Soc., Vol. 362, Number 4 (2010), 20532087.

[8] V. Tотік, Polynomial inverse images and polynomial inequalities, Acta Math., 187(2001), 139-160. 